RESSOURCES HUMAINES

PAR LIONEL DIONNE

\title{
La loyauté dans les relations hiérarchiques
}

Depuis les trente dernières années, la théorie de l'échange supérieur/subalterne (ESS) a évolué. Cet échange est maintenant mesuré en utilisant une approche multidimensionnelle plutôt qu'unidimensionnelle. Les quatre dimensions utilisées pour mesurer I'ESS sont I'affection, la loyauté, la contribution et le respect professionnel. La présente recherche est basée sur des données provenant de dix-neuf banques dans la région des Maritimes du Canada. Elle veut démontrer qu'il existe un lien entre l'ESS et la satisfaction à l'emploi des subalternes. Les résultats indiquent que seule la dimension loyauté réussit à prédire la satisfaction à l'emploi de façon significative.

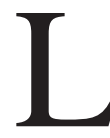

a théorie de l'échange supérieur/subalterne (ESS) porte sur les interactions entre le supérieur et les subalternes. Elle suggère que dans une organisation, le supérieur n'utilise pas le même style de gestion auprès de tous les subalternes, mais qu'il développe plutôt différents types d'échanges avec chacun d'eux. Dans certains cas, cet échange est affecté uniquement par des clauses contractuelles. Mais dans d'autres situations, l'échange entre les deux parties est caractérisé par la confiance mutuelle, le respect, l'appréciation et l'influence mutuelle (Liden et Maslyn, 1998).

Au fur et à mesure que la relation évolue, la latitude donnée au subalterne par le supérieur s'accentue. D'après Dansereau et al. (1975), la latitude est déterminée par deux éléments : la flexibilité du supérieur à allouer des changements dans la tâche du subalterne ainsi que son inclinaison à utiliser le pouvoir lié à sa position dans le but d'aider le subalterne à résoudre des problèmes reliés à son travail. Cette latitude, qui découle d'un échange plus grand entre le supérieur et le subalterne, mène à une plus grande responsabilisation. Sparrowe (1994) a trouvé une association significative entre l'ESS et la perception du subalterne de la responsabilisation qui lui est accordée. En l'occurrence, l'ESS est associé positi- 
vement à des attitudes favorables, entre autres, la satisfaction à l'emploi. McClane (1991) soutient que les subalternes, dont l'échange est plus grand avec leur supérieur, bénéficient d'une responsabilisation accrue dans l'organisation. Ainsi, leur satisfaction est plus élevée envers leur supérieur, envers la tâche à accomplir, envers leurs coéquipiers de travail, bref, envers leur emploi. Par ailleurs, des recherches suggèrent que la satisfaction du subalterne envers son emploi est affectée par sa capacité de pouvoir négocier une certaine latitude avec son supérieur (Dansereau et al., 1975; Scandura et Grean, 1984).

Les moyens utilisés pour évaluer la qualité de l'ESS ont évolué à travers les années. Des études antécédentes utilisaient une échelle de mesure unidimensionnelle pour tenter d'expliquer les comportements des supérieurs envers les subalternes. Cependant, une telle approche ne permettait pas de saisir la multiplicité des rôles dans l'organisation. Actuellement, une échelle de mesure multidimensionnelle, comprenant quatre dimensions, permet de discriminer avec plus de précision la complexité des interactions entre le supérieur et les subalternes. Ces dimensions, sont l'affection, la loyauté, la contribution et le respect professionnel (Liden et Maslyn, 1998).

La présente étude a pour but d'analyser la relation entre l'ESS et la satisfaction à l'emploi auprès des employés de banques. Puisque l'utilisation de l'échelle de mesure multidimensionnelle développée par Liden et Maslyn (1998) permet d'étudier simultanément différentes dimensions de l'échange entre le supérieur et les subalternes, cette étude devrait permettre de déterminer le pouvoir prédictif de chacune de ces dimensions en relation avec la satisfaction à l'em- ploi des employés. Depuis plusieurs années, la satisfaction à l'emploi est un domaine de recherche qui intéresse de nombreux chercheurs. Des études ont abordé diverses variables reliées à la satisfaction au travail, mais relativement peu l'ont fait par le biais de la théorie de l'ESS, tel qu'il est actuellement conceptualisé.

\section{1. Études préliminaires de la théorie de l'ESS}

Northouse (1997) rapporte qu'avant l'étude de l'ESS, le leadership était considéré comme une approche unidimensionnelle. On pensait alors que le supérieur agissait de la même façon avec tous les subordonnés. Par exemple, il aurait eu un style de gestion qu'il appliquait de façon collective envers tout le groupe. Contrairement à cette présomption, la théorie de l'ESS met en évidence les différences pouvant exister dans les relations d'un supérieur avec chacun de ses subalternes.

Selon Liden, Sparrowe et Wayne (1997), l'étude de l'ESS est issue de la théorie des rôles (Kahn et al., 1964) qui soutient que les subalternes d'une organisation accomplissent leur travail en adoptant un rôle ou des comportements qui sont attendus d'eux par ceux qui détiennent un pouvoir hiérarchique. La théorie de l'ESS a été décrite pour la première fois dans les études de Dansereau et al. (1975), Graen et Cashman (1975) et Graen (1976). Depuis, plusieurs changements ont modifié cette théorie (Yukl, 1998).

En étudiant les relations des dyades formées par un supérieur avec chacun de ses subalternes, les chercheurs ont établi qu'il existe deux types généraux de relations (Graen, 1976). Le premier type de relation est circonscrit par les descriptions d'em- 
ploi et les ententes contractuelles, alors que le deuxième type est caractérisé par la qualité des rapports entre le supérieur et les subalternes. Par ce deuxième type de relation, le supérieur a un pouvoir d'influence et il peut ainsi jouer un rôle non négligeable dans la satisfaction à l'emploi de chacun de ses subalternes. Selon Dansereau et al. (1975), les subalternes qui ont une relation positive avec le supérieur reçoivent plus d'informations, d'échanges personnels et d'attention positive de la part de ce dernier. De plus, le supérieur les perçoit comme étant des subalternes plus fiables et plus impliqués. De façon implicite, ils sont considérés comme faisant partie d'un groupe privilégié. Par ailleurs, les subalternes qui ne font pas partie de ce groupe ont moins d'affinité avec le supérieur. Celui-ci les perçoit comme étant des subalternes qui sont moins fiables et moins impliqués dans leur emploi.

\section{Forces de la théorie de l'ESS}

Selon Northouse (1997), la théorie de l'ESS apporte une contribution appréciable à la compréhension du leadership. Il souligne quatre forces de cette théorie.

Premièrement, la théorie de l'ESS reconnaît que, dans l'organisation, certains subalternes apportent une plus grande contribution que d'autres.

Une deuxième force de la théorie de l'ESS se remarque par le fait qu'elle est la seule approche de l'étude du leadership qui permet de placer la relation dyadique au centre du processus de leadership.

Une autre force de la théorie de l'ESS est qu'elle accorde de l'importance à la qualité de la communication dans le leadership. La communication permet à chacun de créer et de soutenir des échanges efficaces lors- qu'elle est caractérisée par la confiance, le respect et l'engagement mutuels entre les deux parties.

Une dernière force de la théorie de l'ESS, soulignée par les recherches, est que sa mise en application a des conséquences positives au sein des organisations. Graen et Uhl-Bien (1995) précisent que la théorie de l'ESS est associée à des aspects positifs pour les organisations, tels que l'amélioration du niveau de performance, du climat de travail et des comportements des employés, ou encore à l'augmentation de l'engagement personnel et la responsabilisation.

\section{Critiques de la théorie de l'ESS}

Selon Burns et Otte (1999), en raison de sa nouveauté relative, la théorie de l'ESS n'est pas encore assez bien développée pour devenir un guide pour les dirigeants en ressources humaines. Ils soutiennent que des recherches supplémentaires sont nécessaires afin de rendre cette théorie plus opérationnelle, en particulier en ce qui a trait aux moyens d'améliorer les relations entre le supérieur et les subalternes, lorsque celles-ci ne sont pas positives.

Une autre critique de cette théorie vient du fait que les chercheurs utilisent différentes échelles de mesure pour évaluer la qualité de l'ESS. Par exemple, Graen et Uhl-Bien (1991) mesurent la qualité de l'ESS par une échelle unidimensionnelle comprenant sept items, alors que Liden et Maslyn (1998) utilisent une échelle multidimensionnelle, comptant douze items pour capter la qualité de l'ESS. Ceci rend le travail des chercheurs plus difficile car les comparaisons des résultats d'une étude à l'autre doivent être effectuées sous beaucoup de réserves. 


\section{Développement de l'échelle LMX-MDM}

Liden et Maslyn (1998) ont développé une échelle de mesure multidimensionnelle appelée LMX-MDM. Cette échelle est utilisée pour mesurer l'ESS à partir de quatre différentes dimensions qui sont: l'affection, la loyauté, la contribution et le respect professionnel. L'affection se définit comme étant l'attraction mutuelle des membres d'une dyade basée sur leur relation interpersonnelle plutôt que sur le travail ou les valeurs professionnelles. $\mathrm{La}$ loyauté est le témoignage de support public pour les buts et les caractéristiques de l'autre membre de la dyade. La contribution est une perception du niveau actuel d'activité reliée au travail que chaque membre de la dyade fournit pour l'atteinte d'un but commun. Enfin, le respect professionnel dénote la perception du degré auquel chaque membre de la dyade a bâti une réputation, à l'intérieur ou à l'extérieur de l'organisation, d'exceller dans son champ de travail.

\section{Multidimensionnalité de l'ESS}

Dienesch et Liden (1986) ont été parmi les premiers auteurs à développer une échelle de mesure multidimensionnelle afin d'apporter une clarté au niveau théorique ainsi qu'une plus grande précision au niveau de la recherche empirique. Ils ont catégorisé la théorie de l'ESS en trois dimensions, la dimension affection, la dimension loyauté et la dimension contribution. Plus tard, une quatrième dimension, proposée par Liden et Maslyn (1998), est venue enrichir l'échelle de mesure développée par Dienesch et Liden. Il s'agit de la dimension respect professionnel.

\section{I. - ÉNONCÉ DU PROBLÈME}

Selon Dansereau et al. (1975) et Sparrowe (1994), la qualité de l'ESS a un effet sur la satisfaction à l'emploi chez les subalternes. La qualité de l'ESS entre un supérieur et un subalterne est affectée par chaque membre de la dyade. Dans l'étude du leadership, plusieurs auteurs choisissent d'étudier les caractéristiques des supérieurs. Cependant, étant donné que le subalterne joue lui aussi un rôle de premier plan dans l'établissement de l'ESS, il semble important de se pencher sur ses perceptions en ce qui à trait à la qualité de la relation qu'il entretient avec son supérieur ainsi que sur sa satisfaction à l'emploi.

Actuellement, l'échelle de mesure développée par Liden et Maslyn (1998) semble être un instrument adéquat permettant d'étudier l'ESS par une approche multidimensionnelle. En plus de tracer un portrait global décrivant la qualité de l'ESS entre le supérieur et ses subalternes, elle permet de mettre chacune des dimensions identifiées en relation avec des aspects importants de la gestion, par exemple, la satisfaction à l'emploi chez les subalternes.

La présente étude a pour but de d'analyser la relation entre la qualité de l'ESS et la satisfaction à l'emploi des subalternes. Plus précisément, elle tente de répondre aux questions suivantes:

1. Quelle est la qualité de l'ESS et quel est le degré de satisfaction à l'emploi chez les subalternes?

2. Est-ce que l'affection, la loyauté, la contribution et le respect professionnel sont des dimensions qui sont en relation avec la satisfaction à l'emploi chez les subalternes? 
3. Quel est l'effet de l'affection, la loyauté, la contribution et le respect professionnel sur la satisfaction à l'emploi chez les subalternes?

\section{Variables de l'étude}

Deux variables sont nécessaires pour répondre aux questions de la recherche. La première, la variable indépendante, est la qualité de l'échange entre les supérieurs et les subalternes (ESS). Cette variable est mesurée à l'aide des 12 énoncés du questionnaire LMX-MDM. Elle permet de connaître le degré de latitude dont dispose le subalterne dans sa relation avec son supérieur. La seconde variable, la variable dépendante, est la satisfaction à l'emploi des subalternes, mesurée par le Minnesota Satisfaction Questionnaire (MSQ).
Selon Schriesheim, Neider et Scandura (1998), il y a une forte corrélation entre la responsabilisation, la productivité et la satisfaction à l'emploi. Plus le degré de l'ESS entre un supérieur et un subalterne est élevé, plus élevé sera le degré de satisfaction du subalterne. Les résultats de la présente étude devraient donc présenter une corrélation positive entre la latitude des subalternes, telle que mesurée par le questionnaire sur l'ESS et la satisfaction à l'emploi. Par ailleurs, le questionnaire LMX-MDM permet de mesurer quatre dimensions de la relation entre le supérieur et le subalterne: l'affection, la loyauté, la contribution et le respect professionnel. Ceci devrait permettre d'analyser l'importance de chacune de ces dimensions pour prévoir la satisfaction à l'emploi des subalternes.

\section{II. - MÉTHODOLOGIE}

\section{1. Échantillonnage et choix des sujets}

Puisque les succursales des banques sont relativement nombreuses et faciles d'accès, parce qu'elles ont des caractéristiques semblables quel que soit l'endroit où elles sont situées et parce que les rôles des supérieurs et des subalternes y sont bien définis et comparable d'une succursale à l'autre, la collecte des données a été effectuée dans ce type d'établissement. En tout, 19 succursales ont été ciblées. Il s'agit de succursales de la région des Maritimes au Canada.

Pour les fins de la présente étude, des instruments de mesure ont été distribués à des subalternes par le biais de leur supérieur. Chaque supérieur a été contacté personnellement, par une rencontre de 20 à 30 minutes, afin de permettre au chercheur de fournir des indications verbales, en plus des indications écrites, concernant la distribution et l'administration des instruments de mesure et pour répondre à toutes autres questions portant sur la présente recherche. Des questionnaires furent distribués auprès de 276 caissières et caissiers, aux services de 19 succursales bancaires différentes. De ce nombre, 158 ont complété les instruments de mesure, les ont retournés par la poste, dans une enveloppe pré-adressée et pré-affranchie, ce qui représente un retour de $57 \%$. 


\section{Instruments et échelles de mesure}

Deux questionnaires ont été utilisés dans cette recherche.

- Le premier, le questionnaire LMX-MDX est une échelle de mesures de l'ESS, développée par Liden et Maslyn (1998). Cet instrument a été utilisé pour mesurer l'échange supérieur/subalterne, tel que perçue par les subalternes. Cette échelle de mesure comporte 12 énoncés représentant les quatre dimensions de l'ESS identifiées par les auteurs, c'est-à-dire, l'affection, la loyauté, la contribution et le respect professionnel. Trois énoncés portent sur chacune des dimensions. Dans l'ensemble, les participants doivent répondre aux 12 énoncés à l'aide d'une échelle de mesure de type Likert comprenant 7 points leur permettant ainsi d'indiquer leur degré d'accord avec chacun des énoncés. Puisque cette échelle de mesure a été administrée dans sa langue d'origine, c'est-à-dire l'anglais, auprès de participants anglophones, il est possible de se fier aux qualités psychométriques de cet instrument.

- Le second instrument de mesure utilisé pour la présente étude fut le Minnesota Satisfaction Questionnaire (Weiss et al., 1967), qui comporte 20 énoncés portant sur la satisfaction à l'emploi. Selon les auteurs, ce questionnaire mesure la satisfaction selon différents aspects du travail et selon l'environnement de travail. Il rend possible l'obtention d'un rendement individuel de la satisfaction selon les raisons individuelles de chaque répondant. Ce questionnaire comporte deux sections. La première comporte 12 énoncés permettant de mesurer la satisfaction intrinsèque et la seconde comporte six énoncés permettant de mesurer la satisfaction extrinsèque. Pour y répondre, les participants disposent d'une échelle de mesure de type Likert de cinq points permettant d'exprimer leur degré de satisfaction.

\section{Résultats}

Les résultats de la recherche sont présentés sous forme de tableaux. En premier lieu, viennent les données descriptives permettant de répondre à la première question de la recherche. Par la suite, un tableau de corrélation présente l'information nécessaire pour répondre à la deuxième question. Finalement, des résultats obtenus à des analyses de régression linéaires multiples apportent une réponse à la troisième question de la recherche. La discussion des résultats s'effectue dans le même ordre logique.
La première question à l'étude avait pour but de mettre en évidence la qualité de l'ESS et le degré de satisfaction à l'emploi chez les subalternes. Le tableau 1, qui rapporte les résultats obtenus à l'échelle de mesure LMX-MDM, permet de constater le degré de latitude donné par les supérieurs, tel que perçu par les subalternes. Les moyennes obtenues à chacun des énoncés par l'ensemble des participants sont indiquées dans ce tableau. Les participants ont pu exprimer leur degré d'accord à chaque énoncé sur une échelle de type Likert comprenant 7 points, allant du négatif au positif. Les énoncés 1 à 3 déterminent la dimen- 
sion affection, les énoncés 4 à 6 représentent la dimension loyauté, les énoncés 7 à 9 désignent la dimension contribution et les énoncés 10 à 12 reflètent la dimension respect professionnel. Afin de faciliter l'analyse, un indice, qui est en fait la combinai- son des questions se rapportant à chaque dimension, à été calculé pour chacune d'entre elle. Ces moyennes sont les suivantes: affection $(5,41)$, loyauté $(5,03)$, contribution $(5,67)$ et le respect professionnel $(5,64)$.

Tableau 1

MESURES DE L'ESS SELON LIDEN ET MASLYN (1998)

\begin{tabular}{|c|c|c|c|c|c|c|c|c|}
\hline \multicolumn{9}{|c|}{$\begin{array}{c}\text { Tableau } 1 \\
\text { MESURES DE L’ESS SELON LIDEN ET MASLYN (1998) }\end{array}$} \\
\hline & & ale & & & & $\begin{array}{l}\text { men } \\
\text { cord }\end{array}$ & & Moyenne \\
\hline 1. J'aime mon supérieur comme personne. & 1 & 2 & 3 & 4 & 5 & 6 & 7 & 5,69 \\
\hline $\begin{array}{l}\text { 2. Mon supérieur est le type d'individu } \\
\text { qu'on aimerait avoir comme ami. }\end{array}$ & 1 & 2 & 3 & 4 & 5 & 6 & 7 & 5,40 \\
\hline $\begin{array}{l}\text { 3. J'ai beaucoup de plaisir à travailler avec } \\
\text { mon supérieur. }\end{array}$ & 1 & 2 & 3 & 4 & 5 & 6 & 7 & 5,13 \\
\hline $\begin{array}{l}\text { 4. Mon supérieur défend mes actions } \\
\text { à son supérieur même s'il ne connaît pas } \\
\text { tous les faits sur un problème. }\end{array}$ & 1 & 2 & 3 & 4 & 5 & 6 & 7 & 4,66 \\
\hline $\begin{array}{l}\text { 5. Mon supérieur viendrait à mon secours si } \\
\text { j'étais «attaqué » par d'autres }\end{array}$ & 1 & 2 & 3 & 4 & 5 & 6 & 7 & 5,08 \\
\hline $\begin{array}{l}\text { 6. Mon supérieur me défendrait devant les } \\
\text { autres dans l'organisation si j'avais fait } \\
\text { une faute honnête. }\end{array}$ & 1 & 2 & 3 & 4 & 5 & 6 & 7 & 5,35 \\
\hline $\begin{array}{l}\text { 7. Je fais du travail pour mon supérieur qui } \\
\text { dépasse ma description d'emploi. }\end{array}$ & 1 & 2 & 3 & 4 & 5 & 6 & 7 & 5,27 \\
\hline $\begin{array}{l}\text { 8. Je suis prêt à faire un effort supplémentaire, } \\
\text { au-delà de ce qui est habituellement requis, } \\
\text { afin d'aider mon supérieur à atteindre ses } \\
\text { objectifs. }\end{array}$ & 1 & 2 & 3 & 4 & 5 & 6 & 7 & 5,82 \\
\hline $\begin{array}{l}\text { 9. Ça ne me dérange pas de travailler plus fort } \\
\text { pour mon supérieur. }\end{array}$ & 1 & 2 & 3 & 4 & 5 & 6 & 7 & 5,91 \\
\hline $\begin{array}{l}\text { 10. Je suis impressionné des connaissances } \\
\text { de mon supérieur dans son emploi. }\end{array}$ & 1 & 2 & 3 & 4 & 5 & 6 & 7 & 5,62 \\
\hline $\begin{array}{l}\text { 11. Je respecte les connaissances et les } \\
\text { compétences de mon supérieur dans son emploi. }\end{array}$ & 1 & 2 & 3 & 4 & 5 & 6 & 7 & 5,72 \\
\hline $\begin{array}{l}\text { 12. J'admire les habiletés professionnelles } \\
\text { de mon supérieur. }\end{array}$ & 1 & 2 & 3 & 4 & 5 & 6 & 7 & 5,58 \\
\hline
\end{tabular}

Affection (Q1, Q2, Q3), loyauté (Q4, Q5, Q6), contribution (Q7, Q8, Q9), respect professionnel (Q10, Q11, Q12). 
Ces résultats permettent de constater que la loyauté est la dimension qui obtient l'indice le moins élevé auprès des participants à cette étude. Toutefois, une moyenne de 5,03 se situe au-dessus de la moyenne de l'échelle de 1 à 7 , ce qui porte à croire que les subalternes croient que leur supérieur serait enclin à leur témoigner un support public en ce qui à trait à leur travail ou à leurs caractéristiques. D'autre part, un indice de 5,67 à la dimension «contribution» reflète la meilleure moyenne pour les quatre dimensions. $\mathrm{Ce}$ résultat dénote que les subalternes ayant participé à cette étude sont d'avis qu'ils contribuent de façon importante pour atteindre les buts visés par le supérieur, et par le fait même, par l'organisation. En d'autres termes, ils ont le sentiment de fournir des efforts supplémentaires afin de supporter le supérieur dans l'atteinte des objectifs communs. Pour ce qui est de la dimension «affection», avec un indice de 5,41 , il semble que les subalternes apprécient leurs supérieurs. Cette affection est basée sur les relations interpersonnelles et non pas sur les valeurs professionnelles. Enfin, une moyenne de 5,64 en ce qui à trait à la dimension respect professionnel dénote que les subalternes sont enclins à respecter les qualités professionnelles de leurs supérieurs.

Les résultats du Minnesota Satisfaction Questionnaire sont présentés dans le tableau 2. On y retrouve les 20 énoncés représentant le degré de satisfaction des subalternes. Douze de ces énoncés représentent la satisfaction intrinsèque (énoncés $1,2,3,4,7,8,9,10,11,15,16$ et 20) et six représentent la satisfaction extrinsèque (énoncés 5, 6, 12, 13, 14 et 19). Les énoncés 1 à 20 représentent la satisfaction générale. Les participants ont exprimé leur degré de satisfaction sur une échelle Likert de cinq points allant du négatif au positif. $\mathrm{La}$ moyenne globale aux énoncés permettant d'évaluer la satisfaction intrinsèque est de 4,03 . Pour ce qui est de la satisfaction extrinsèque, la moyenne aux énoncés correspondants est de 3,42. Quant à la satisfaction générale, c'est-à-dire, l'ensemble des 20 énoncés, la moyenne est de 3,84.

Ces résultats suggèrent que les subalternes trouvent une satisfaction intrinsèque dans l'accomplissement de leur travail. Cependant, en ce qui a trait aux facteurs externes, par exemple, le style de gestion de leur supérieur, les politiques de l'entreprise ou la reconnaissance pour un travail bien fait, ils sont indécis quant à leur degré de satisfaction. De façon générale, les subalternes ayant participé à l'étude ont tendance à se dire satisfaits de leur emploi.

La deuxième question de l'étude était la suivante: Est-ce que l'affection, la loyauté, la contribution et le respect professionnel sont des dimensions qui sont en relation avec la satisfaction à l'emploi chez les subalternes? Le tableau 3 indique les corrélations entre chacune des dimensions et la satisfaction générale à l'emploi. 


\begin{tabular}{|c|c|c|c|c|c|c|}
\hline \multicolumn{7}{|c|}{$\begin{array}{c}\text { Tableau } 2 \\
\text { MESURES DE LA SATISFACTION À L'EMPLOI } \\
\text { (Minnesota Satisfaction Questionnaire) }\end{array}$} \\
\hline \multicolumn{7}{|c|}{ Dans mon présent emploi, voici comment je me sens par rapport à: } \\
\hline & $\mathrm{TI}$ & $\mathrm{I}$ & $\mathrm{N}$ & $\mathrm{S}$ & TS & Moyenne \\
\hline 1. Être capable de me tenir occupé tout le temps & 1 & 2 & 3 & 4 & 5 & 4,31 \\
\hline 2. La chance de travailler seul & 1 & 2 & 3 & 4 & 5 & 4,23 \\
\hline 3. La chance de faire différentes choses & 1 & 2 & 3 & 4 & 5 & 4,14 \\
\hline 4. La chance d'être quelqu'un dans la communauté & 1 & 2 & 3 & 4 & 5 & 3,91 \\
\hline 5. La façon que mon supérieur gère ses subalternes & 1 & 2 & 3 & 4 & 5 & 3,82 \\
\hline $\begin{array}{l}\text { 6. La compétence de mon supérieur à prendre } \\
\text { des décisions }\end{array}$ & 1 & 2 & 3 & 4 & 5 & 4,07 \\
\hline $\begin{array}{l}\text { 7. Être capable de faire des choses qui ne vont } \\
\text { pas à l'encontre de ma conscience }\end{array}$ & 1 & 2 & 3 & 4 & 5 & 4,19 \\
\hline 8. La sécurité d'emploi & 1 & 2 & 3 & 4 & 5 & 3,89 \\
\hline 9. La chance de faire des choses pour les autres & 1 & 2 & 3 & 4 & 5 & 4,37 \\
\hline $\begin{array}{l}\text { 10. La chance de dire aux autres ce qu'ils } \\
\text { doivent faire }\end{array}$ & 1 & 2 & 3 & 4 & 5 & 3,61 \\
\hline $\begin{array}{l}\text { 11. La chance de faire quelque chose qui mette } \\
\text { mes habiletés à contribution }\end{array}$ & 1 & 2 & 3 & 4 & 5 & 4,08 \\
\hline $\begin{array}{l}\text { 12. La façon que les politiques de l'entreprise } \\
\text { sont mises en application }\end{array}$ & 1 & 2 & 3 & 4 & 5 & 3,61 \\
\hline 13. Mon salaire et la quantité de travail effectuée & 1 & 2 & 3 & 4 & 5 & 3,24 \\
\hline 14. Les chances d'avancement dans mon emploi & 1 & 2 & 3 & 4 & 5 & 3,28 \\
\hline 15. La liberté d'utiliser mon propre jugement & 1 & 2 & 3 & 4 & 5 & 3,961 \\
\hline $\begin{array}{l}\text { 16. La chance d'essayer mes propres méthodes } \\
\text { pour faire les choses }\end{array}$ & 1 & 2 & 3 & 4 & 5 & 3,81 \\
\hline 17. Les conditions de travail & 1 & 2 & 3 & 4 & 5 & 4,08 \\
\hline 18. La collaboration de mes collègues entre eux/elles & 1 & 2 & 3 & 4 & 5 & 4,04 \\
\hline 19. Les félicitations suite à travail bien fait & 1 & 2 & 3 & 4 & 5 & 3,76 \\
\hline 20. Le sens d'accomplissement obtenu dans mon job & 1 & 2 & 3 & 4 & 5 & 3,89 \\
\hline
\end{tabular}

$\mathrm{TI}$ : très insatisfait avec cet aspect de mon emploi. $\mathrm{I}$ : insatisfait avec cet aspect de mon emploi. $\mathrm{N}$ : je ne peux pas décider entre satisfait ou non avec cet aspect de mon emploi. S: satisfait avec cet aspect de mon emploi. TS : très satisfait avec cet aspect de mon emploi. 


\begin{tabular}{|c|c|c|c|c|c|c|c|c|}
\hline \multicolumn{9}{|c|}{$\begin{array}{c}\text { Tableau } 3 \\
\text { MOYENNES, ÉCARTS TYPES ET CORRÉLATIONS } \\
\text { DE L'ESS ET DE LA SATISFACTION }\end{array}$} \\
\hline \multirow[b]{2}{*}{ Variables } & \multicolumn{8}{|c|}{ Intercorrelations } \\
\hline & 1 & 2 & 3 & 4 & 5 & 6 & 7 & 8 \\
\hline \multicolumn{9}{|l|}{ L'ESS } \\
\hline 1. Affection & & & & & & & & \\
\hline 2. Loyauté &, $83 * *$ & & & & & & & \\
\hline 3. Contribution &, $59 * *$ &, $57 * *$ & & & & & & \\
\hline 4. Respect professionnel &, $70 * *$ &, $73 * *$ &, $60 * *$ & & & & & \\
\hline 5. L'ESS &, $93 * *$ &, $93 * *$ &, $79 * *$ &, $80 * *$ & & & & \\
\hline \multicolumn{9}{|l|}{ Satisfaction } \\
\hline 6. Intrinsèque &, $40 * *$ &, $45 * *$ &, $33 * *$ & $.32 * *$ &, $45 * *$ & & & \\
\hline 7. Extrinsèque &, $60 * *$ &, $63 * *$ &, $34 * *$ &, $51 * *$ &, $60 * *$ &, $70 * *$ & & \\
\hline 8. Satisfaction générale &, $53 * *$ &, $57 * *$ &, $35 * *$ &, $44 * *$ &, $55 * *$ &, $93 * *$ &, $90 *$ & \\
\hline Moyenne & 5,41 & 5,03 & 5,66 & 5,38 & 5,38 & 4,03 & 3,63 & 3,91 \\
\hline Écart type & 1,47 & 1,50 & 1,21 & 1,48 & 1,23 &, 58 &, 85 & ,61 \\
\hline
\end{tabular}

$* *<0,01 . \mathrm{n}=15 \%, \mathrm{n}=158$. Échelle de l'ESS allant de 1 à 7 . Échelle de satisfaction allant de 1 à 5.

Les résultats de la présente étude permettent de constater qu'il y a effectivement une relation entre l'affection, la loyauté, la contribution, le respect professionnel et la satisfaction à l'emploi chez les subalternes. Chaque dimension est reliée à la satisfaction. De plus, la qualité de l'ESS dans son ensemble est aussi reliée à la satisfaction. Ces résultats confirment donc les études de Liden et Maslyn (1998) qui mettent en évidence des relations entre chacune des dimensions et la satisfaction à l'emploi.

La troisième question de cette recherche s'intéressait à connaître l'effet de chacune des quatre dimensions: l'affection, la loyauté, la contribution et le respect professionnel sur la satisfaction à l'emploi chez les subalternes. Afin de vérifier le pouvoir prédictif des quatre dimensions mention- nées, des analyses de régression ont été effectuées par une méthode d'élimination progressive. Le tableau 4 présente les trois équations qui ont été analysées afin de connaître la contribution de chacune des dimensions. Il est possible de constater, en considérant la première équation (colonnes 1-3), que la dimension loyauté contribue de façon importante à la satisfaction à l'emploi. Cette dimension prédit la satisfaction avec un $\mathrm{R}^{2}$ de, 32 , ce qui suggère que la dimension loyauté est la variable pouvant prédire avec plus de justesse la satisfaction dans cette première équation. On peut donc conclure qu'une seule dimension contribue de façon significative à ce résultat. Il s'agit de la loyauté. Dans les deux autres résultats, l'affection et le respect professionnel ont un apport minime et non significatif. 


\begin{tabular}{|c|c|c|c|c|c|c|c|c|c|}
\hline \multicolumn{10}{|c|}{$\begin{array}{c}\text { Tableau } 4 \\
\text { VARIABLES PRÉDICTIVES DE LA SATISFACTION } \\
\text { À L'EMPLOI DES SUBALTERNES }(\mathrm{N}=158)\end{array}$} \\
\hline & \multicolumn{3}{|c|}{$\mathrm{R}^{2}=, 32$} & \multicolumn{3}{|c|}{$\mathrm{R}^{2}=, 33$} & \multicolumn{3}{|c|}{$\mathrm{R}^{2}=, 33$} \\
\hline Variables prédictives & $\beta$ standardisé & SE & $\rho$ & $\beta$ standardisé & $\mathrm{SE}$ & $\rho$ & $\beta$ standardisé & SE & $\rho$ \\
\hline Loyauté &, 568 & ,027 & ,000 & ,426 & ,048 &, 000 &, 412 & ,052 &, 002 \\
\hline Affection & & & & ,170 &, 049 & $\mathrm{~ns}$ & ,160 & 051 & $\mathrm{~ns}$ \\
\hline Respect professionnel & & & & & & & ,032 & ,041 & $\mathrm{ns}$ \\
\hline
\end{tabular}

Dans la présente recherche, la dimension loyauté est, sans contredit, celle qui est le plus en mesure de prédire la satisfaction à l'emploi chez les subalternes. Ce résultat suggère l'importance pour les gestionnaires de porter une attention particulière au témoignage de support public envers les subordonnés en ce qui concerne les buts et les caractéristiques de l'entreprise.

\section{CONCLUSION}

La présente recherche ajoute un élément nouveau, celui de vérifier la contribution de chacune des dimensions de l'ESS pour prédire la satisfaction à l'emploi des subalternes. En effectuant simplement une étude corrélationnelle entre l'ESS et le niveau de satisfaction général des subalternes, il aurait été possible d'identifier une relation positivement significative d'une ampleur de ,55 entre ces deux variables. Cependant, il n'aurait pas été possible de savoir quelle dimension de l'ESS présente le meilleur pouvoir prédictif de la satisfaction à l'emploi.

Les présents résultats confirment que les dimensions affection, loyauté, contribution et respect professionnel sont en relation avec la satisfaction à l'emploi chez les subalternes. Toutefois, les analyses de régression ont permis de constater que les quatre dimensions ont des pouvoirs prédictifs particuliers. Selon cette recherche, c'est surtout la dimension loyauté qui prédit la satisfaction à l'emploi. La loyauté est le témoignage de support public pour les buts et les caractéristiques de l'autre membre du dyade de l'ESS. La loyauté implique une fidélité de l'individu qui est généralement constante d'une situation à une autre. Le gestionnaire qui voudra satisfaire davantage ses subalternes ne devra pas négliger cet aspect particulier de l'ESS.

Ces nouvelles connaissances contribuent de façon importante au développement de l'étude de l'ESS. De plus, les répondants pour la présente recherche proviennent tous du milieu bancaire, ce qui peut permettre la généralisation des résultats. Dans des recherches éventuelles, il serait intéressant de refaire l'exercice avec un nombre plus élevé de candidats provenant de différents milieux. Afin de réaliser des analyses statistiques plus développées, il serait aussi important de recueillir des informations auprès des deux membres de chaque dyade, c'est-à-dire, les supérieurs et les subalternes. 


\section{BibLIOGRAPHIE}

J. Z. Burns, F. L. Otte, "Implications of Leader-Member Exchange Theory and Research for Human Resource Development Research", Human Resource Development Quarterly, ${ }^{\circ} 10$, 1999, p. 225-247.

F. Dansereau, G. Graen, et W. J. Haga., "A vertical dyad linkage approach to leadership within formal organizations: A longitudinal investigation of the role making process", Organizational Behavior and Human Performance, $\mathrm{n}^{\circ}$ 13, 1975, p. 46-78.

Dienesch R.M., Liden R.C., "Leader-member exchange model of leadership: A critique and further development", Academy of Management Review, $\mathrm{n}^{\circ}$ 11, 1986, p. 618-634.

G. B. Graen, "Role-making process within complex organizations", Handbook of industrial and organizational psychology, M.D. Dunnette (ed.), Chicago, Rand McNally, 1976, p. 1201-245.

G. B. Graen, M. Uhl-Bien, "The Transformation of Professionals Into Self-Managing and Partially Self Designing Contributors", Journal of Management Systems, $\mathrm{n}^{\circ}$ 3, 1991, p. 25-39.

G. B. Graen, M. Uhl-Bien, "Relationship-Based Approach to Leadership. Development of Leader-Member Exchange LMX Theory”, Leadership Quarterly, n 6, 1995, p. 219-247.

G. B. Graen, J. Cashman, "A role-making model of leadership in formal organizations: A developmental approach, Leadership frontiers, J. G. Hunt et L. L. Larson (eds), Kent State University Press, 1975, p. 143-165.

R. L. Kahn, D. M. Wolfe, R. P. Quinn, J. D. Snowk, et R. A., Rosenthal, Organizational stress: Studies in role conflict and ambiguity, Wiley, New York, 1964.

R. C. Liden, R. T. Sparrowe, S. J. Wayne, "Leader-Member Exchange Theory. The past and Potential for the Future", Research in Personnel and Human Resources Management, G. Ferris (ed.), Jai Press, Greenwich Ct., 1997, p. 47-119.

R. C. Liden, J. M. Maslyn, "Multidimensionality of Leader-Member Exchange: An Empirical Assessment Through Scale Development", Journal of Management, $\mathrm{n}^{\circ}$ 24, 1998, p. 3-72.

M. McClane, E. William, "Implications of Member Role Differentiation, Analysis of a Key Concept in the LMX Model of Leadership", Group et Organization Studies, n 16, 1991, p. 102-113.

P. G. Northouse, "Leadership. Theory and Practice", Sage Publications, Thousand Oaks, ch. 7., 1997, p. 109-129.

R. A. Scandura, G. B. Graen, "Moderating effects of initial leader-member exchange status on the effects of a leadership intervention", Journal of Applied Psychology, n 69, 1984, p. 428-436.

C. A. Schriesheim., L. L. Neider, T. A. Scandura, "Delegation and Leader-Member Exchange: Main Effects, Moderators, and Measurement issues", Academy of Management Journal, $\mathrm{n}^{\circ}$ 41, 1998, p. 298-318.

R. T. Sparowe, "Empowerment in the hospital industry: An exploration of antecedents and outcomes", Hospitality Research Journal, n 17, 1994, p. 51-73. 
Weiss D. J., Dawis R. V., England G.W., Lofquist L. H., "Manual for the Minnesota Satisfaction Questionnaire", University of Minnesota, 1967.

G. Yukl, Leadership in Organizations, $4^{\text {th }}$ edition, Prentice Hall, Upper Saddle River, NJ, ch. 7, 1998, p. 149-174. 